\title{
GENETIC ASSOCIATION OF LEUCKOCYTES LEVELS DURING THE ACUTE PHASE OF STROKE
}

Nuria P Torres-Aguila (1), Caty Carrera (2), Anne-Katrine Giese (3), Elena Lopez-Cancio (4), Antoni Davalos (4), Jordi Jimenez-Conde (5), Jaume Roquer (5), Natalia Cullell (1), Elena Muiño (1), Jara Cárcel-Márquez (1), Jonathan Gonzalez-Sanchez (1,6), Cristòfol Vives-Bauza (7), Jerzy Krupinski (8), Laura Heitsch (9), Laura Ibañez (10), Carlos Cruchaga (10), Natalia Rost (3), Joan Montaner (2,11), Jin-Moo Lee (12), Israel Fernandez-Cadenas $(1,13)$.

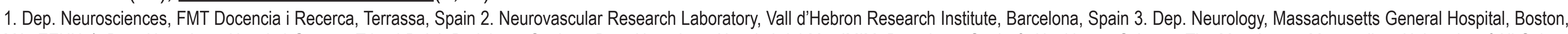

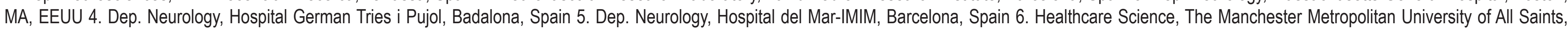

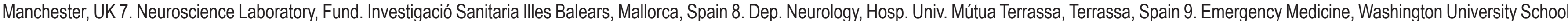

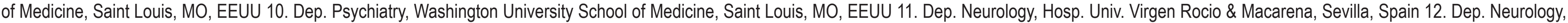
Washington University School of Medicine, Saint Louis, MO, EEUU 13. Stroke Pharmacogenomics and Genetics, Sant Pau Institute of Research, Barcelona Spain. e-mail: israelcadenas@yahoo.es

\section{BACKGROUND \& OBJECTIVE}

Several genes are associated with stroke risk, however, little is known about stroke outcome. Neutrophils and other immune cells have a key role in inflammation during the acute phase of stroke and have been associated with poor functional and neurological outcome.
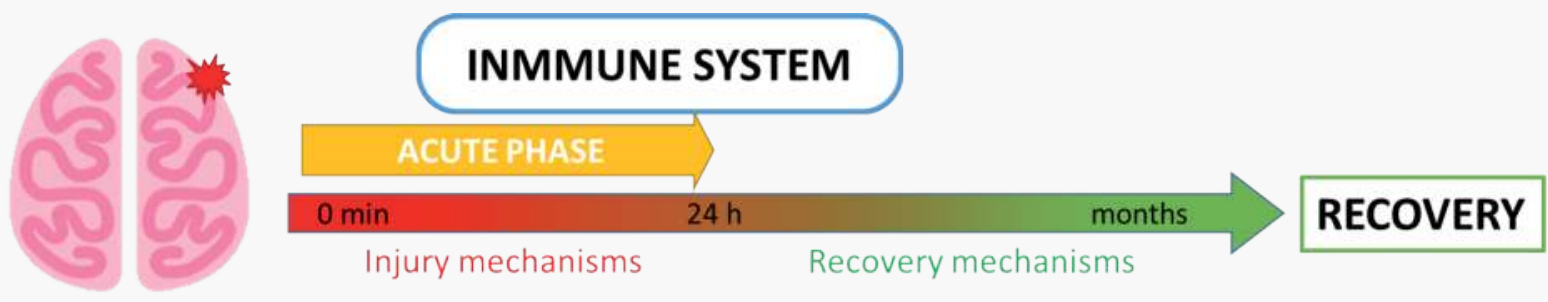

Our aim is to find genetic risk factors associated with neutrophils levels (NL) and white blood cells counts (WBCc) during the acute phase of ischemic stroke.

\section{RESULTS - WBCC}

A total of 1575 patients were analyzed for WBCc and 5.271.103 SNPs pass QCs. We found a Genome-Wide suggestive associations for WBCc on Chr4 (Top-SNP p-value $=4.3 e-07$ ).

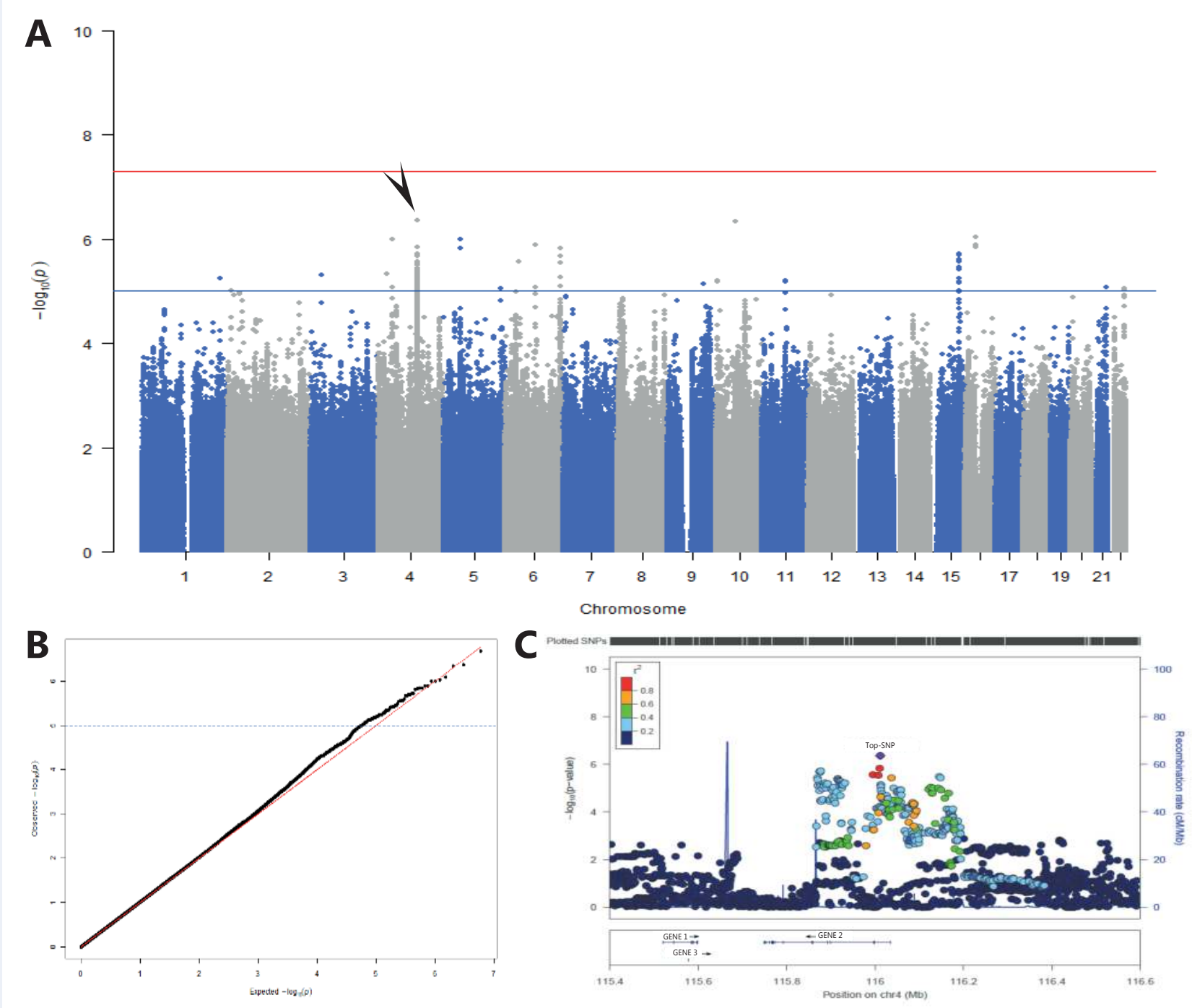

A) Manhattan plot of GWAS results for WBCc. Blue line: suggestive threshold $(p=1 e-05)$; Red line: genome-wide threshold $(p=5 e-08)$; Black arrow: top-hit. B) QQplot of WBCc GWAS results. Blue line: suggestive threshold. C) Locus Plot of Chr4 hit. Color scale: Linkage Disequilibrium (LD) between Top-SNP and other SNPs. P-values are expressed as -log10(P-value).

\section{CONCLUSIONS}

We have found new genetic associations with NL and WBCc during the acute phase of stroke. These SNPs were not previously associated with leukocytes levels in healthy population, suggesting a different modulation of immune cells during the acute phase of stroke.

\section{ACKNOWLEDGMENTS}

This project was funded by the Instituto de Salud Carlos III (Generation project), Red Retics (INVICTUS plus), and European comission (FEDER) Part of the project was founded by the NIH (GENISIS project).

\section{METHODS}

Patients from 8 different cohorts with data of NL and WBCc during the acute phase ( $<24 \mathrm{~h}$ stroke onset) were included in the study. The phases of the study were: Genotyping, Imputation, SNP association analysis and Metanalysis.

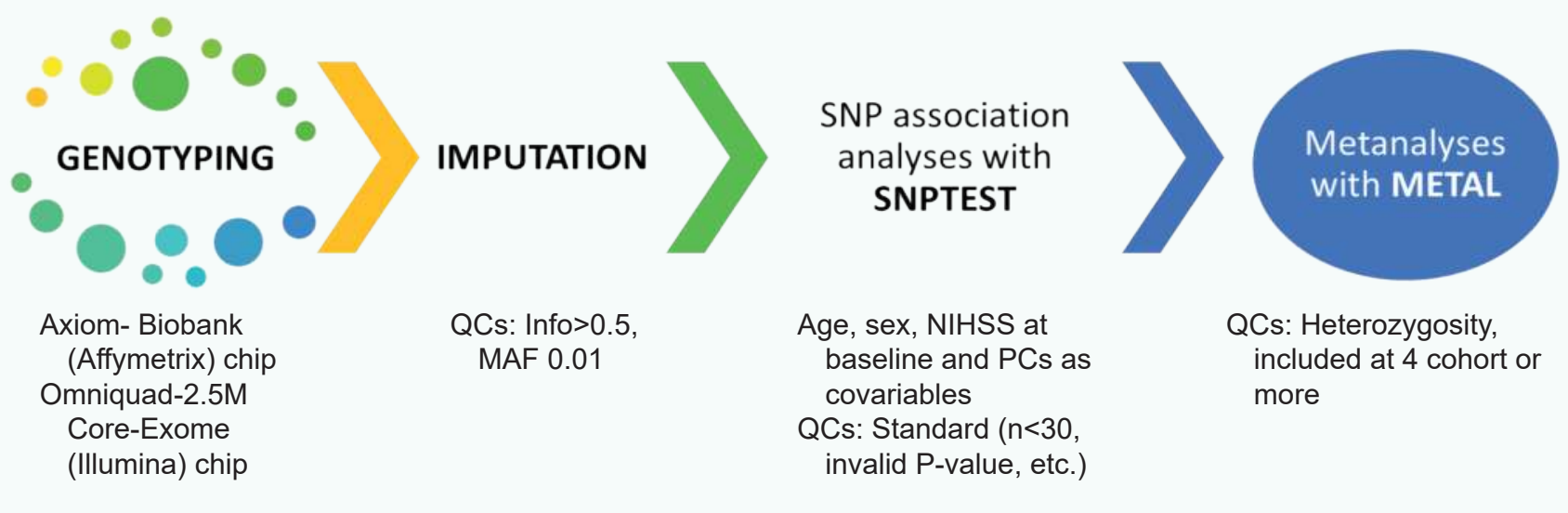

\section{RESULTS - NL}

A total of 941 patents were analyzed for NL and 4.430.759 SNPs pass QCs. We found a Genome-Wide suggestive associations for NL on Chr6 (Top-SNP p-value $=2.2 \mathrm{e}-07$ ).

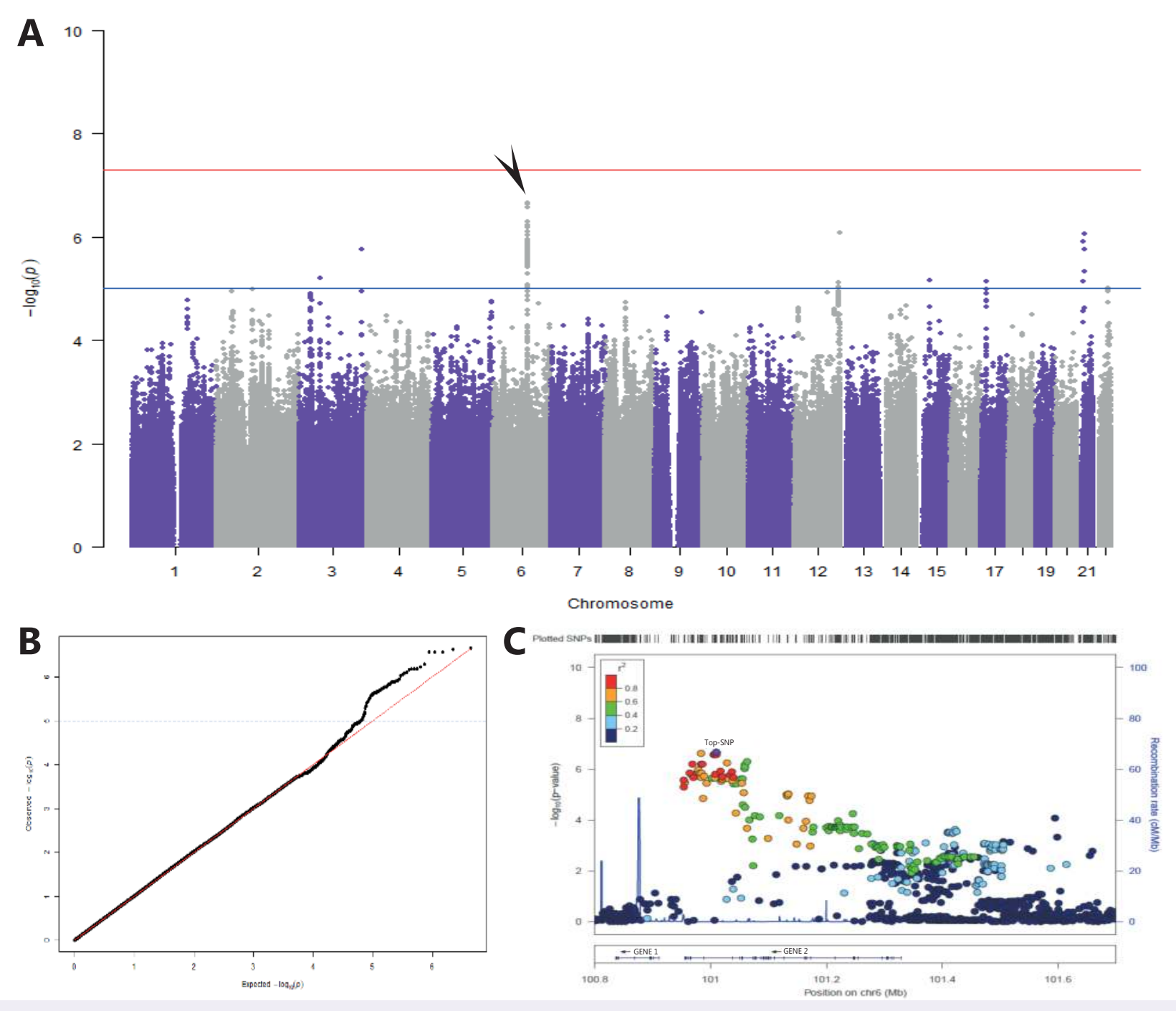

A) Manhattan plot of GWAS results for NL. Blue line: suggestive threshold $(p=1 e-05)$; Red line: genome-wide threshold ( $p=5 e-08)$; Black arrow: top-hit. B) QQplot of NL GWAS results. Blue line: suggestive threshold. C) Locus Plot of Chr6 hit. Color scale: Linkage Disequilibrium (LD) between Top-SNP and other SNPs. P-values are expressed as -log10(P-value).

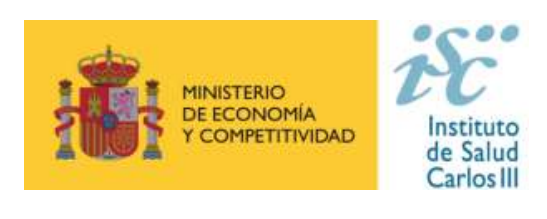

UNIVERSITAT $T_{D E}$ BARCELONA

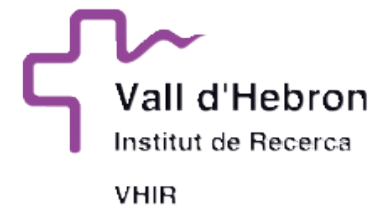
VHIR
Fundació Docència i Recerca MútuaTerrassa 